\title{
Eosinophilic enteritis presenting as a perforated duodenal ulcer
}

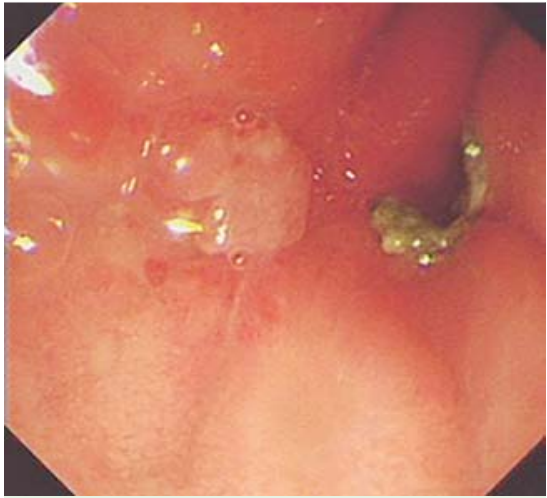

Fig. 1 Esophagogastroduodenoscopy showing gastritis and a duodenal ulcer with stenosis.

Eosinophilic gastroenteritis (EGE) is a rare condition of unknown etiology that is characterized by eosinophilic infiltration in the layers of the gastrointestinal tract [1]. It can affect any part of the gastrointestinal tract but most commonly affects the stomach $[1,2]$. This report describes a rare case of EGE presenting as a perforated duodenal ulcer with subsequent duodenal stenosis.

A 26-year-old man was referred to us with duodenal obstruction following a laparotomy for a perforated duodenal ulcer. He had undergone repair of the ulcer and 2 weeks later had presented with vomiting and weight loss. Endoscopy showed gastritis and a duodenal ulcer with stenosis ( Fig. 1). A barium meal showed narrowing at the first part of the duodenum ( $\bullet$ Fig. 2). He was given treatment for Helicobacter pylori and pantoprazole. A repeat endoscopy 2 months later showed a deformed pylorus, prepyloric nodular mucosa, and an almost circumferential duodenal ulcer with significant narrowing.

Biopsies from the ulcer showed a marked eosinophilic infiltrate diagnostic of EGE ( $\bullet$ Fig. 3). He received prednisolone as a tapered course over 1 month and pantoprazole, after which, there was a marked improvement in his symptoms. A repeat endoscopy showed a healed duodenal ulcer and a postbulbar stricture ( $\bullet$ Fig. 4 ). Central radial expansion balloon dilation was performed. He received another course of steroids and 1 month later was well and gaining weight with no vomiting. Talley et al. suggested three diagnostic criteria for EGE: (i) gastrointestinal symp-
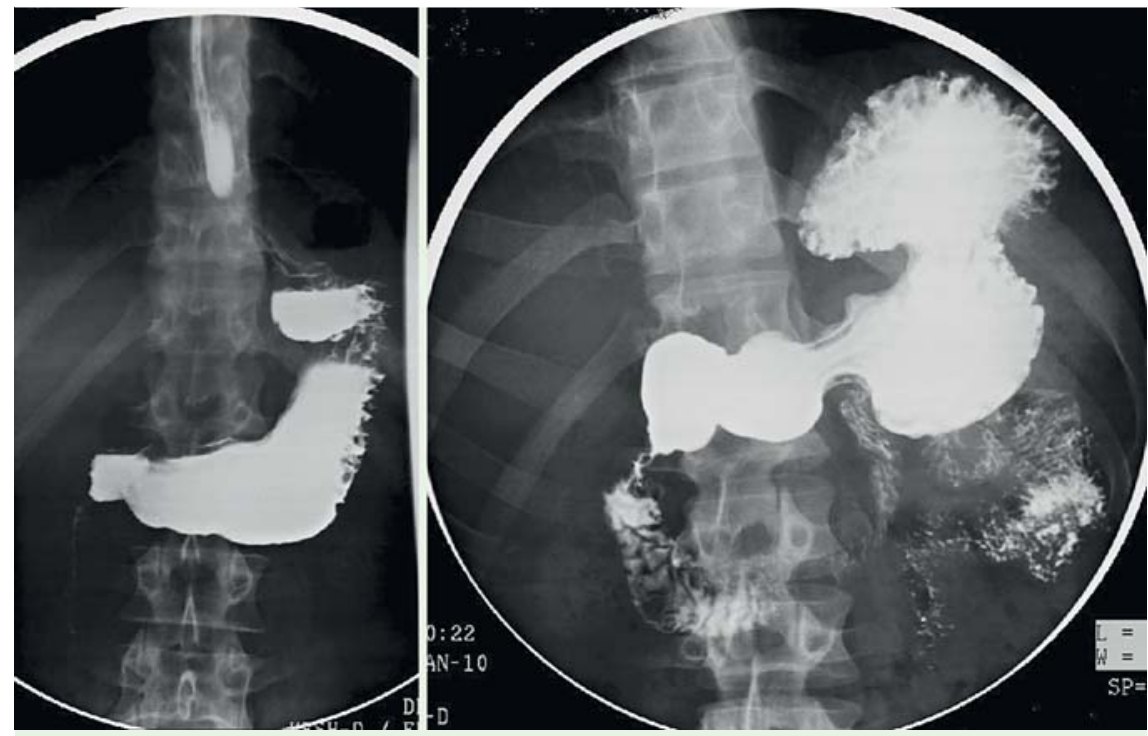

Fig. 2 Barium meal showing a short segment of narrowing in the first part of the duodenum with peripheral ulceration suggesting chronic ulceration with secondary fibrotic changes and subsequent narrowing.
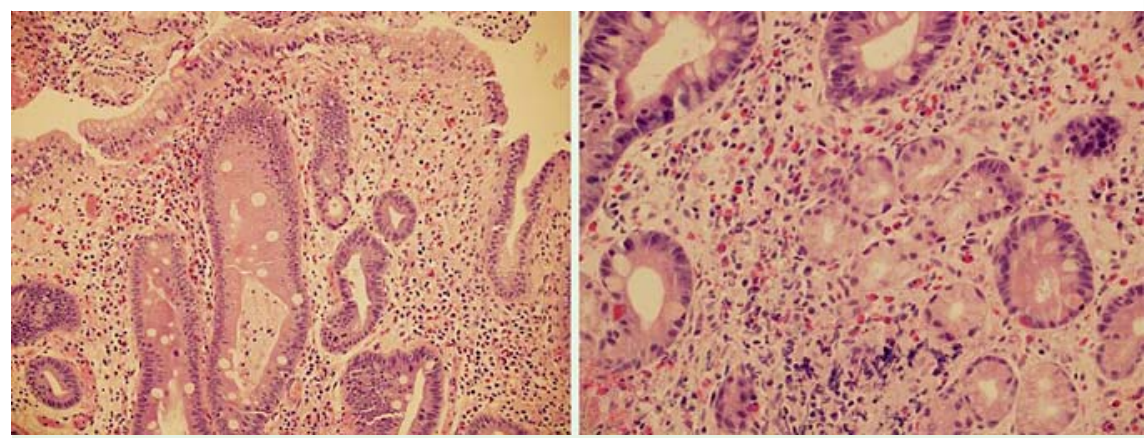

Fig. 3 Biopsies from the duodenal ulcer showing a marked eosinophilic infiltrate, diagnostic of eosinophilic gastroenteritis (EGE).

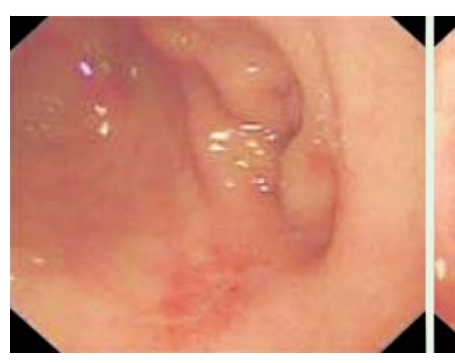

toms; (ii) demonstration of eosinophilic infiltration in the gastrointestinal tract, or presence of high eosinophil count in fluid; (iii) no evidence of parasitic or extraintestinal disease [3]. EGE has been classified, depending on the extent of bowel wall involvement, into mucosal, muscular, and serosal $[1,3]$. In the past, peptic ulceration accounted for most cases of gastric outlet obstruction. Nowadays, other causes such as malignancy and EGE must be excluded.

EGE may also present acutely $[4,5]$ as was the case for our patient who presented with peritonitis secondary to a perforated peptic ulcer, with a subsequent stricture 
that was presumed to be postsurgical but proved to be due to EGE. To the best of our knowledge, this is the first report of EGE presenting as a perforated duodenal ulcer.

Endoscopy_UCTN_Code_CCL_1AB_2AD_3AC

Competing interests: None

H. Issa ${ }^{1}$, B. Bseiso ${ }^{1}$, A. H. Al-Salem ${ }^{2}$

1 Department of Internal Medicine, King Fahad Specialist Hospital, Dammam, Saudi Arabia

2 Department of Pediatric Surgery, Maternity and Children Hospital, Dammam, Saudi Arabia

\section{References}

1 Khan S, Orenstein SR. Eosinophilic gastroenteritis. Gastroenterol Clin N Am 2008; 37: 333-348

2 Chen MJ, Chu CH, Lin SC et al. Eosinophilic gastroenteritis: clinical experience with 15 patients. World J Gastroenterol 2003; 9: 2813-2816

3 Talley NJ, Shorter RG, Phillips SF, Zinsmeister $A R$. Eosinophilic gastroenteritis: a clinicopathological study of patients with disease of the mucosa, muscle layer, and subserosal tissues. Gut 1990; 31: 54-58

4 Blanco-Guerra C, Cazana JL, Villas F et al. Ileal perforation due to eosinophilic gastroenteritis. Am J Gastroenterol 1991; 86: 16891690

5 Maeshima A, Murakami H, Sadakata H et al. Eosinophilic gastroenteritis presenting with acute pancreatitis. J Med 1997; 28: 265 272
Bibliography

DOI $10.1055 / \mathrm{s}-0030-1256526$

Endoscopy 2011; 43: E358 -E359

(c) Georg Thieme Verlag KG Stuttgart · New York . ISSN 0013-726X

Corresponding author

\section{A. H. Al-Salem, MD}

Department of Pediatric Surgery, Maternity and Children Hospital,

Dammam

P.O. Box 61015

Qatif 31911

Saudi Arabia

ahsalsalem@hotmail.com 